\title{
Medical Therapy of Active Ulcerative Colitis
}

\author{
Martin Bürger ${ }^{\mathrm{a}}$ Carsten Schmidt $^{\mathrm{a}}{\text { Niels } \text { Teich }^{\mathrm{b}} \text { Andreas Stallmach }}^{\mathrm{a}}$ \\ a Department of Internal Medicine IV, Jena University Hospital, Jena, Germany \\ ${ }^{b}$ Group Practice for Digestive and Metabolic Diseases, Leipzig, Germany
}

\section{Keywords}

Ulcerative colitis - Medical therapy - Innovations .

Anti-TNF · Vedolizumab · Fecal microbiota transfer

\section{Summary}

Background: Medical therapy of mild and moderate ulcerative colitis (UC) of any extent is evidence-based and standardized by national and international guidelines. However, patients with steroid-refractory UC still represent a challenge. Methods: A literature search using PubMed (search terms: ulcerative colitis, therapy, new, 1-2008-2015) resulted in 821 publications. For the current article, 88 citations were extracted including 36 randomized controlled studies, 18 reviews, and 8 meta-analyses. Results: In steroid-refractory UC, early intensive therapy using anti-tumor necrosis factor (TNF) antibodies or the calcineurin inhibitors cyclosporine and tacrolimus is indicated in any case to prevent progression to a toxic megacolon and/or to avoid proctocolectomy. In patients with chronic disease activity, treatment with anti-TNF antibodies has a higher level of evidence than azathioprine therapy and should therefore be preferred. However, there is a subgroup of UC patients who may achieve prolonged steroid-free remission on azathioprine monotherapy. The importance of vedolizumab, a newly registered inhibiting antibody against integrin, has not yet been fully clarified since direct comparison studies are lacking, in particular in relation to anti-TNF antibodies. Conclusion: There is a great need for additional innovative therapies, especially in cases of primary non-response or secondary loss of response to anti-TNF antibodies. New small molecules (Janus kinase inhibitors) are promising with an acceptable safety profile and efficacy in UC. Further, strategies that target the intestinal microbiome are currently considered for patients with active or relapsing UC, and may in the future open up new therapeutic options.

(C) 2015 S. Karger GmbH, Freiburg

\section{Introduction}

Although the etiopathogenesis of ulcerative colitis (UC) remains elusive, substantial progress in the understanding of the development and progression of UC as well as the establishment of effective treatment strategies has been achieved in the past decades (fig. 1). The clinical course of UC is highly variable and ranges from a single episode to potentially life-threatening continuous disease. Prospective population-based studies have described different types of long-term disease courses: patients with a decrease in disease intensity following the first flare-up (44\%), patients with an increase in intensity (3\%), patients with chronic continuous symptoms (24\%), and patients with chronic intermittent symptoms (29\%) [1]. Long-term prognosis studies conducted in Copenhagen County have previously indicated that approximately $50 \%$ of the UC cohort at any given time will be in remission, whereas $50 \%$ will relapse within 1 year. Approximately $15-20 \%$ of UC patients experience acute severe flare-ups which are serious and potentially life-threatening $[2,3]$.

The treatment of UC is sequential and tailored to the individual patient, because no one treatment is universally effective for everyone ('one size does not fit all'). Hence, we will first focus on standard situations which can be treated easily using a classical step-up concept. The mainstay in the treatment of mild to moderate UC are 5-aminosalicylates (5-ASA; mesalamine) which are highly effective as a topical treatment (suppositories, enemas, foams) in patients with distal UC; oral formulations in the form of various commercial modified-release products are effective in the treatment of mild to moderately active UC and in preventing relapse. Corticosteroids are indicated for more severe disease where mesalamine has limited efficacy in inducing remission. Second, we discuss treatment options in steroid-refractory patients as well as the difficult issue of 'rescue' therapy in patients with severe UC. Especially in this group, early introduction of immunosuppressive drugs (calcineurin inhibitors or anti-tumor necrosis factor (TNF)- $\alpha$ antibodies) may have an impact on the disease course during severe episodes including the development of a toxic mega-

\section{KARGER}

Fax +497614520714

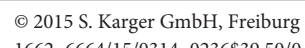

Prof. Dr. med. Andreas Stallmach Klinik für Innere Medizin IV Friedrich-Schiller-Universität Jena Erlanger Allee 101, 07740 Jena, Germany andreas.stallmach@med.uni-jena.de 


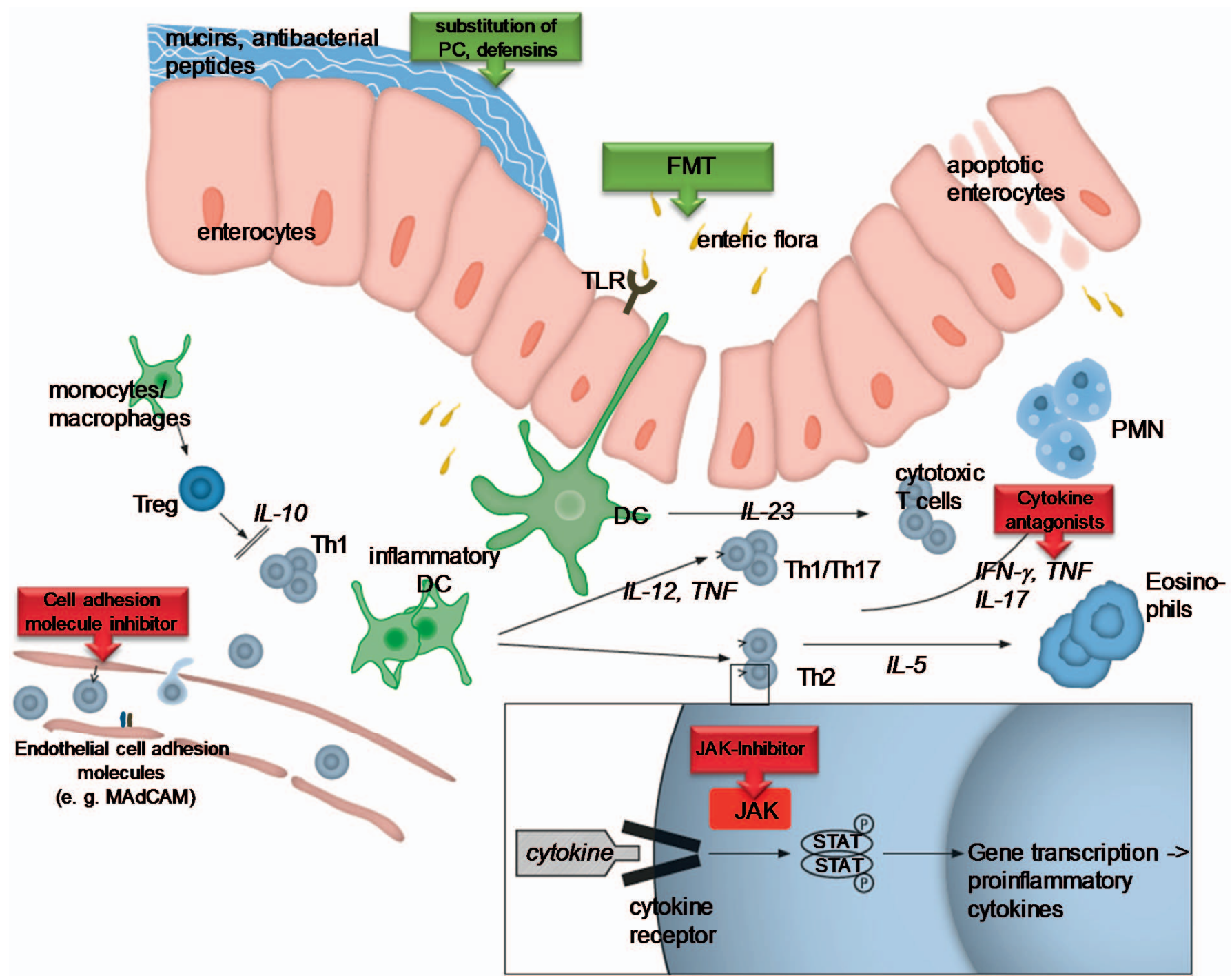

Fig. 1. Physiopathology of ulcerative colitis. Under normal conditions, the intestinal homeostasis is regulated by several mechanisms. Disturbance of this balance triggers the chronic inflammatory process found in ulcerative colitis. During early inflammation, an increasing barrier defect with uncontrolled uptake of foreign luminal antigens (bacteria, bacterial products) leads to the activation of different innate immune cells located in the intestine, including natural killer cells, mast cells, neutrophils, macrophages, and dendritic cells (DC). An excessive, uncontrolled inflammatory reaction promotes further the activation of the adaptive immune response. Abnormally activated effector $\mathrm{CD} 4^{+} \mathrm{T}$ helper $(\mathrm{Th})$ cells synthetize and release different inflammatory mediators that generate the vicious circle of inflammation that leads to chronic tissue injury and epithelial damage.

Red arrows indicate blockade of proinflammatory mechanisms, green arrows indicate substitution or replacement of protective agents like phosphatidylcholine (PC) or beneficial bacteria by fecal microbiota transfer (FMT). IL = Interleukin; Treg = regulatory T cells; TLRs = Toll-like receptors; PMNs = polymorphonuclear leukocytes.

colon. However, it must be pointed out that in these cases of acute severe UC, a high 1-year colectomy rate of up to $50 \%$ should be anticipated [4-6].

\section{Activity-Adapted Therapy in Standard Situations - Patients with Mild to Moderate Activity}

Recent guidelines concerning the medical treatment and management of active UC recommend an activity-adapted approach. Treatment decisions should be based on disease activity and distribution (proctitis, left-sided, extensive colitis) [7]. It is important to note that all treatment decisions should be planned and discussed in detail with the patient. For a successful therapy, patient compliance is critical [8], which has been shown in several studies; a significant amount of UC flare-ups are caused by a lack of patient adherence, especially when complicated or uncomfortable treatment regimens are used [9]. Therefore, the importance of choice of therapy and consecutive patient compliance needs to be addressed to avoid unsatisfactory responses to treatment and relapses caused by non-adherence [10].

The first clinical consideration in the management of patients with active UC is to confirm disease activity and exclude alternative diagnoses. During disease flare-ups in particular gastrointesti- 
nal infections must be excluded; especially assays for Clostridium difficile and cytomegalovirus infection should be requested in view of the rising incidence and association of these infections with increased mortality in patients with UC. If active colitis is considered as the main cause of symptoms, therapy should be induced promptly. There is a plethora of different clinical scoring systems used in clinical trials; however, for daily practice it is most important to differentiate between patients with mild or moderate activity and those with severe UC. Patients with severe disease should be hospitalized. Hospitalization should be considered for all patients who have more than 6-10 bloody stools per day, associated with fever, tachycardia, or an increase in erythrocyte sedimentation rate (ESR) according to the criteria of Truelove and Witts [11].

\section{Mild to Moderate Activity in Proctitis}

If mild to moderate inflammation is limited to the rectum, topical treatment with mesalamine is the first-line therapy. Marshall et al. [12] confirmed the superiority of this treatment in inducing remission. The preferred treatment is 1-g mesalamine suppositories once daily, since Andus et al. [13] demonstrated non-inferiority of this approach to divided doses. Additionally, there is no dose response above $1 \mathrm{~g}$ mesalamine per day. Topical steroids can be used as second-line therapy since topical mesalamine is superior to rectal corticosteroids [14], or as an alternative for patients with intolerance to topical mesalamine [15]. Safdi et al. [16] postulated that combined topical and oral mesalamine treatment seems to be more effective; however, this study included patients with leftsided colitis also. Currently, there is a lack of studies for only proctitis treated with a combination of oral and topical mesalamine. A combination of topical mesalamine and topical steroid appears to be more effective than either agent alone [17]. Patients who fail to respond to the regimens above require additional treatment with oral prednisolone.

\section{Left-Sided Colitis}

The recommended treatment for mild to moderate distal/leftsided UC is a combination of topical mesalamine enemas/foams $1 \mathrm{~g} /$ day and oral mesalamine $>2 \mathrm{~g} /$ day $[7,15]$. Combined therapy showed significantly higher efficacy in disease improvement and led to faster improvement of rectal bleeding $[16,18]$. Furthermore, topical mesalamine in comparison to oral mesalamine alone showed a higher rate of mucosal absorption [19], which supports the concept of combined therapy since the therapeutic effect of mesalamine correlates with its mucosal concentration [20]. Topical therapy foam enemas and liquid enemas are seen as equivalent treatment options for inducing remission [21]; using low volume enemas might result in better patient compliance [22]. An equivalent alternative to rectal mesalamine may be rectal beclomethasone dipropionate [23]. Similar to topical rectal medication, single oral doses of mesalamine are non-inferior to divided doses per day [24] and should improve patient adherence [25]. Different from that is the dose response to oral mesalamine: concerning induction of remission, Ford et al. [26] showed in their meta-analysis evidence that doses of $>2 \mathrm{~g}$ /day are more effective than doses of $<2 \mathrm{~g} /$ day. The ASCEND trial even showed a benefit of double doses with 4.8 $\mathrm{g}$ /day at week 6 concerning mucosal healing and induction of remission, although there was no further benefit at week 8 [27]. Hence, the European guideline recommends at least $2 \mathrm{~g}$ mesalamine per day [7]. The use of systemic steroids needs to be addressed at the latest 14 days after treatment failure.

\section{Extensive Colitis}

The basic therapy recommendation for extensive mild to moderate UC is similar to that for distal or left-sided colitis [15]. A combined therapy using oral and topical 5-ASA medication is superior to single use of either. Also, there is no significant difference in the induction of remission concerning various 5-ASA formulations, although mesalamine is better tolerated $[28,29]$. This is different for patients with limited distribution of UC where use of systemic corticosteroids should be considered earlier for those with extensive colitis and/or treatment failure and especially for patients already receiving immunomodulatory therapy [30].

\section{Severe Ulcerative Colitis of Any Extent}

Severe UC is characterized by systemic disease activity with severe intestinal symptoms, well described by the easy-to-use Truelove and Witt criteria [11]. To provide an appropriate therapy for severe UC, patients generally require hospitalization, preferably at a specialized center with experience in treating high-risk patients; this reduces mortality to as little as $1 \%$ [31]. In these cases, steroid therapy is still the gold standard [32]. Severe acute UC should be treated with an initial intravenous dose of corticosteroids of $1 \mathrm{mg} /$ $\mathrm{kg}$. While there are no data supporting a possible benefit of using doses above $60 \mathrm{mg} /$ day [33], doses of less than $15 \mathrm{mg}$ /day are considered ineffective [34]. If no improvement is seen or symptoms are worsening after $72 \mathrm{~h}$, the treatment strategy must be revised. The evaluation of clinical response should be based on comparative and objective laboratory and clinical parameters. If there is a lack of therapy response on day 3 , second-line conventional therapy or proctocolectomy may be considered in patients with steroid-refractory colitis [33]. The perhaps most challenging task for physicians in this scenario is to not miss the time of treatment failure: the time point at which first-line conventional therapy fails and salvage therapy or surgery becomes absolutely necessary. The worst case scenario is an emergency colectomy with a 30-day mortality of up to of 5.2\% [35]. Hence, these patients need to be cared for by a multidisciplinary team of gastroenterologists and colorectal surgeons from the day of admission; under those conditions, mortality rates of less than $1 \%$ and a pooled short-term colectomy rate of $27 \%$ are achievable [33]. 
Theoretically, the term 'refractory colitis' implies an inadequate response to therapy on different levels. Therefore, the terms 'refractory' and 'therapy' require specification. Adequate response should indicate the return to normal bowel function without visible blood or cramping. Most experts equate failure of treatment with salicylates and steroid (topical and systemic) as an inadequate response after 6-8 weeks. In other settings such as acute severe colitis treated with intravenous corticosteroids, the response must be anticipated earlier. As mentioned above, the early therapeutic response to intravenous steroids after 3 days of treatment is a critical determinant of prognosis in acute severe UC. Patients that maintain more than 8 bloody stools per day and patients with 3-8 bloody stools per day and a C-reactive protein (CRP) level of $>45$ $\mathrm{mg} / \mathrm{l}$ are likely to require colectomy. Stool frequency ( $>4$ per day) and elevated CRP levels ( $\geq 25 \mathrm{mg} / \mathrm{l}) 3$ days after the initiation of steroids are independent predictors for poor corticosteroid response and colectomy [36]. Moreover, rectal bleeding and platelet count on day 3 are factors that may predict non-response to intensified corticosteroid treatment [37]. Hypoalbuminemia at onset of therapy, mean stool frequency, and colonic dilatation within the first 3 days of treatment predict the need for short-term surgery. These factors have been combined to a severity score by Ho et al. [38].

\section{Steroid-Refractory Acute Severe Colitis - Calcineurin Inhibitors or Anti-TNF Antibodies?}

As mentioned above, unresponsiveness to intravenous steroid treatment after $72 \mathrm{~h}$ predicts a worse prognosis. To avoid proctocolectomy in these cases, treatment options include the calcineurin inhibitors cyclosporine and tacrolimus as well as the anti-TNF antibodies. Generally, all of these treatment options seem to be similarly effective. The first randomized controlled trial that demonstrated the efficacy of cyclosporine in severe UC [39] included patients who did not respond to the equivalent of $300 \mathrm{mg}$ intravenous hydrocortisone over 3 days with a $\geq 10$ Lichtiger score of an arbitrarily defined clinical activity index. The trial was terminated prematurely after $9 / 11$ (82\%) patients responded to cyclosporine therapy compared to $0 / 9$ patients in the placebo group. Colectomy rates were lower in the cyclosporine treatment group (27\%) than in the placebo group (44\%) [39]. In a second randomized trial, van Assche et al. [40] demonstrated that a lower cyclosporine dose (2 $\mathrm{mg} / \mathrm{kg}$, continuous $24-\mathrm{h}$ infusion) was as effective as $4 \mathrm{mg} / \mathrm{kg}$ and resulted in response rates of more than $80 \%$ after 8 days of treatment. Predictors of short-term cyclosporine failure are quite similar to those of intravenous corticosteroid failure. Signs of systemic inflammation such as body temperature $>37.5^{\circ} \mathrm{C}$, tachycardia, elevated CRP, hypoalbuminemia, and elevated neutrophil band forms at initiation of cyclosporine therapy are predictive for non-response to cyclosporine and subsequent colectomy. Patients with severe endoscopic lesions had to undergo proctocolectomy more frequently than patients without severe endoscopic lesions (for review see [41]). Uncontrolled data demonstrated that the efficacy of tacrolimus in severe steroid-refractory colitis seems to be similar to cyclosporine [42-46]. It is important to note that prophylaxis with sulfamethoxazol/trimethoprim $800 / 160$ for the prevention of pneumocystis pneumonia should be considered for patients undergoing calcineurin inhibitor therapy, especially combination therapies.

In contrast to short-term responses, long-term remission rates after calcineurin inhibitor therapy are conflicting and highly dependent on sufficient maintenance therapy for UC. The majority of published studies and case series report a long-term colectomyfree survival of $40-60 \%$ [47-50]. Thiopurine-naive patients that receive thiopurines after a short-term response to cyclosporine remain free from colectomy in $60-87 \%$ of cases $[51,52]$. These results emphasize the necessity of an effective maintenance therapy in UC patients after a severe flare-up has been successfully treated with calcineurin inhibitors.

Several clinical trials have clearly demonstrated the short-term efficacy of infliximab (IFX; Remicade ${ }^{\circledR}$, Janssen Inc., Toronto, ON, Canada) as the first anti-TNF antibody in avoiding colectomy in patients with acute severe UC. The first positive randomized controlled trial (RCT) that investigated the efficacy and safety of IFX as a rescue therapy for acute steroid-refractory UC was performed in 45 patients with moderate to severe UC between 2001 and 2004 [53]. Patients who received a single dose of IFX were less likely to undergo colectomy within 90 days than placebo patients $(7 / 24$, $29 \%$ vs. $14 / 21,67 \%)$. Retrospective studies on the efficacy of IFX in acute severe colitis demonstrated colectomy rates within 90 days of IFX treatment for severe steroid-refractory UC of 33\% (13/39) in a Scottish cohort [54] and 30\% (17/56) in a Danish cohort [55]. Kohn et al. [56] reported a colectomy-free short-term survival of $70 \%(70 / 83)$ after 2 months. Similar to data on intensified steroid treatment and calcineurin inhibitor rescue therapy, hypoalbumine$\mathrm{mia}<30 \mathrm{~g} / \mathrm{l}$ at admission (odds ratio (OR) 6.9; $\mathrm{p}=0.05$ ) or $<34 \mathrm{~g} / \mathrm{l}$ after 3 days of steroid treatment (OR 12.0; $\mathrm{p}=0.02)$ predicted a nonresponse to IFX and the need for colectomy [54].

Based on this data, which therapeutic approach should be chosen for the individual patient with severe steroid-refractory UC? The various UC therapy trials are difficult to compare. Clinical heterogeneity, incomplete reporting of follow-up data, and different disease-defined endpoints across various clinical studies limit the assignability to individual patients. Therefore, head-to-head RCTs are critical in all areas of clinical medicine, including severe UC. A landmark randomized open-label trial by the GETAID group compared cyclosporine and IFX in UC patients with severe acute flareups after unsuccessful treatment with high-dose intravenous steroids [57]. The vast majority of the included patients (103/111; $93 \%$ ) were thiopurine-naive. Treatment failure occurred in $60 \%$ of patients treated with cyclosporine and in 54\% treated with IFX ( $\mathrm{p}=$ 0.49). Similarly, early response after 7 days ( 85 vs. $86 \%$ ) and colectomy rates after 98 days ( 18 vs. $21 \%$ ) did not differ between the treatment groups. Therefore, an advantage of cyclosporine versus IFX was not confirmed in this first prospective RCT. In another retrospective analysis, the same effectiveness of cyclosporine and IFX therapy in the acute hospital setting was shown, although the 


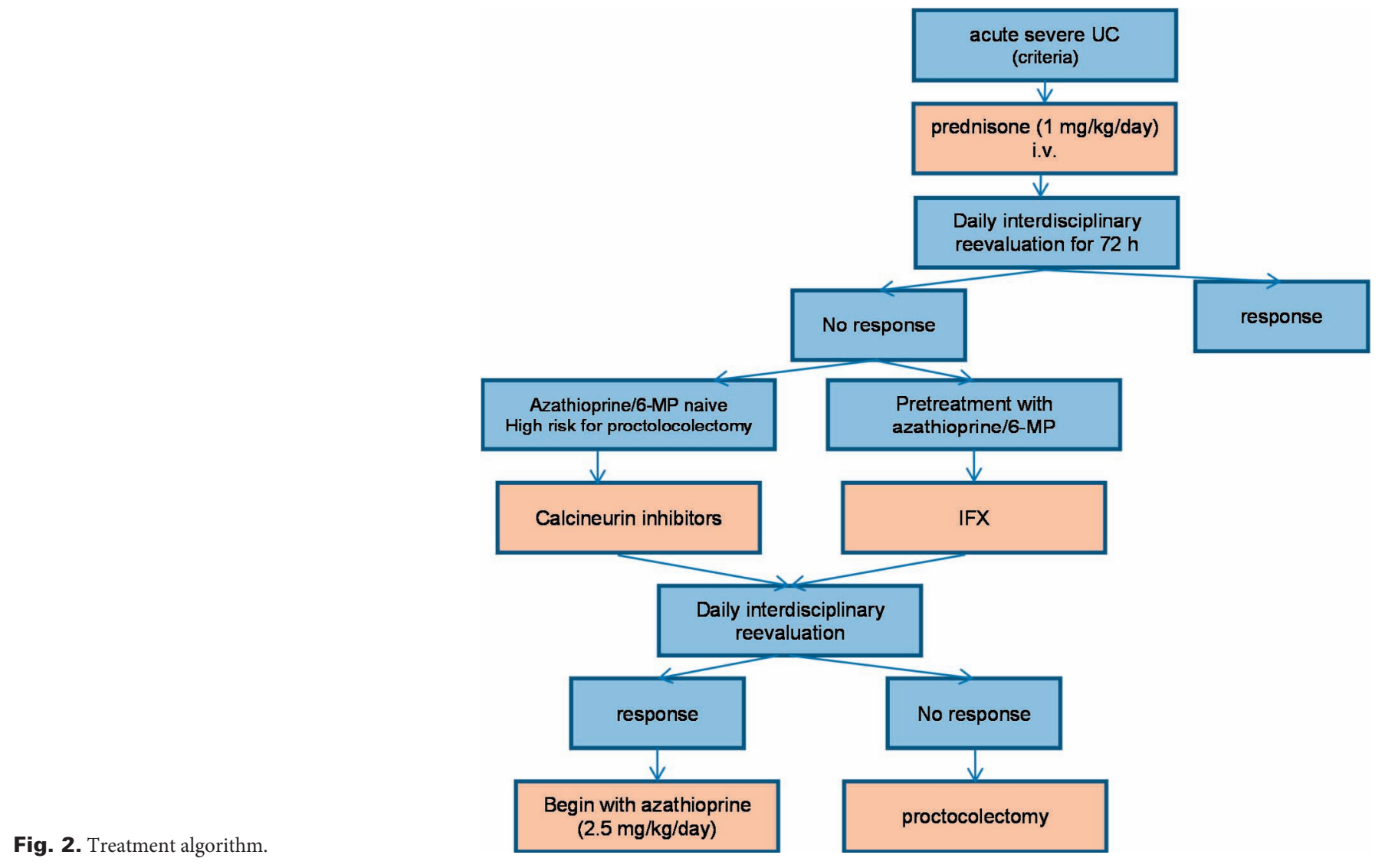

hospital stay for IFX-treated patients was significantly shorter; on the other hand, total treatment costs within the first 3 months were significantly higher [58].

Interestingly, very recently, long-term data comparing both drugs were reported [59]. The median follow-up of the entire cohort was 5.0 years; importantly, azathioprine was started at a dose of $2.5 \mathrm{mg} / \mathrm{kg} /$ day in all patients with clinical response at day 7 . Colectomy-free survival rates at 1,2 , and 5 years were $70 \pm 6 \%, 65$ $\pm 6 \%$, and $61 \pm 7 \%$, respectively, in patients randomized to cyclosporine, and $70 \pm 6 \%, 68 \pm 6 \%$, and $65 \pm 7 \%$, respectively, in those randomized to IFX. Therefore, the prognosis was better than expected and data confirm long-term efficacy and safety profiles of these different treatment strategies and do not favor one drug over the other. This was confirmed by a retrospective analysis from Spain [60]. Overall, the long-term outcome with combined therapy including azathioprine is superior to monotherapy [61].

Thus, for daily practice, the algorithm depicted in figure 2 seems to be an appropriate approach: In patients with acute steroid-refractory UC, treatment intensification should be considered when there is no improvement after $72 \mathrm{~h}$. Patients already undergoing treatment with azathioprine should receive IFX (ensuring longterm remission with continued IFX therapy); azathioprine-naive patients could be treated with cyclosporine. Potential contraindications (e.g., calcineurin-inhibitors: seizures, IFX: abscess-related complications, history of tuberculosis, heart failure grade III/IV) should also be considered. Patients with very high risk (see above) for failure of conservative therapy should be medicated with calcineurin inhibitors due to the much shorter biological half-life.

It is unclear whether the IFX data apply to other anti-TNF antibodies such as adalimumab and golimumab. There are no controlled studies available for adalimumab and golimumab in patients with steroid-refractory UC. In a retrospective multicenter study, Lequoy et al. [62] described remission rates of $63 \%$ after starting adalimumab therapy in patients with refractory colitis. However, the term 'refractory' was not further explained (see above); in addition, it is unclear how many patients had an acute severe flare-up. In a meta-analysis, induction therapy with IFX seemed to be slightly more effective in comparison to the other two antibodies, especially adalimumab [63]. Furthermore, it would be of interest whether the IFX (Remicade) data apply to other infliximab biosimilars in patients with steroid-refractory colitis. Currently, there are no clear recommendations available.

\section{Supportive Therapy in Severe Cases}

Supportive therapy is tremendously important for patients with severe acute UC. Additional treatments like adjusting electrolyte levels (unbalanced electrolyte levels can promote toxic dilatation) [64], prophylactic heparin (UC patients are at higher risk of thromboembolism) [65], blood transfusions, nutritional support, and exclusion of certain medication (non-steroidal anti-inflammatory 
drugs, opioids, etc.) [66] can help reduce complications. Supportive therapy therefore needs to be addressed with every patient admitted to hospital. Antibiotic treatment additional to the routinely given medication did not seem to have an impact on outcome (vancomycin [67], intravenous tobramycin and metronidazole [68, 69]). Therefore, antibiotic treatment should be used based on evidence of bacterial infection.

\section{Innovations}

\section{Vedolizumab for Ulcerative Colitis}

In UC, the inflammatory process is characterized by leukocytic infiltration of the intestinal lamina propria. Inhibition of integrinmediated leukocyte recruitment from the blood to the site of inflammation was therefore selected as a worthwhile target to control the inflammatory cascade [70]. Contrary to an earlier study integrin, i.e. natalizumab, vedolizumab specifically recognizes the $\alpha_{4} \beta_{7}$ integrin and subsequently selectively block gut lymphocyte trafficking without interfering with trafficking to the central nervous system [71]. In two landmark double-blind, placebo-controlled trials of vedolizumab in 895 patients with active UC, response rates with vedolizumab at week 6 were nearly double the rates in the placebo group ( 47 vs. $26 \%$, respectively). After 1 year, more than $40 \%$ of patients who continued to receive vedolizumab were in clinical remission, as compared to $16 \%$ of patients who switched to placebo after a successful vedolizumab induction phase. Furthermore, significantly more patients were in steroid-free remission after 1 year of vedolizumab treatment as compared with placebo. The frequency of adverse events was similar in the vedolizumab and placebo groups, especially with regard to infections and infusion reactions [72].

In Germany within the first year after EMA approval, vedolizumab is usually offered to patients who fail or stop to respond to TNF antagonists or are intolerant to these drugs (own unpublished data). In the GEMINI I trials, however, less than $50 \%$ of patients were previously treated with TNF antagonists. Therefore, the suitable indication for vedolizumab treatment in TNF-naive patients needs to be defined in every day care. In an indirect comparison with the ACT I trial, IFX induction therapy for UC was potentially faster than vedolizumab in the first weeks of therapy [73]. Theoretically, patients with a severe clinical course or cases with steroidrefractory UC could therefore recover faster with a TNF antagonist than with vedolizumab. In contrast, patients with a clinically wellcontrolled but steroid-dependent disease course could be better treated with vedolizumab in the long term. Patients at increased risk for severe infections, i.e. with a history of tuberculosis, cytomegalovirus colitis, asplenia, or elderly patients, could primarily be treated with vedolizumab due to its safety profile. Furthermore, future trials are needed to define the optimal dose, frequency of administration, and long-term efficacy and safety of vedolizumab used for induction and maintenance therapy of UC.

\section{Fecal Microbiota Transplantation in Active Ulcerative Colitis}

The importance of the intestinal microbiome in inflammatory bowel diseases such as UC was one of the key findings of recent years. Several studies demonstrated an imbalance of the normal enteric microbiota composition (termed dysbiosis), which influences the pathophysiology of UC $[74,75]$. For example, a reduction in enteric microbiota diversity has been observed, mainly affecting the anti-inflammatory and/or butyrate-producing bacteria such as Faecalibacterium prausnitzii, Rosebura faecis, and Bacteroides ovatus [76]. Therefore, manipulation of this dysbiosis by employing various strategies including application of pre- and probiotics, antibiotics, or fecal microbiota transplantation (FMT), as an attempt to radically address disturbed microbial composition and diversity, could be an attractive approach for UC patients [77]. While some authors and small case reports/series reported a clear benefit, others could not find a beneficial effect and even saw adverse events in inflammatory bowel disease patients such as fever or diarrhea (for review see [78]). In patients with UC, a randomized controlled study comparing heterologous fecal microbiota with placebo showed positive effects in clinical outcomes [79]. However, very recently, a comparison of transplantation of heterologous and autologous fecal microbiome in patients with mild to moderate UC demonstrated no significant benefit for heterologous FMT [80]. Therefore, to date, there is insufficient data to recommend FMT in active UC.

\section{Future Perspectives}

Without doubt, anti-TNF antibodies have improved the therapy options in patients with UC. In daily clinical practice, there still is a substantial amount of patients either not responding at all to antiTNF antibodies or experiencing a secondary loss of response. The effect of antibodies inhibiting migration of inflammatory cells into the gut mucosa (currently only vedolizumab is approved in Germany) are less efficient in this subgroup of patients with anti-TNF failure.

Beyond the pivotal role of TNFa in the network of proinflammatory mediators, recently several other main components involved in the pathophysiological processes in UC have been elucidated that may serve as therapeutic targets. Subsequently, new classes of drugs have been developed for the treatment of patients suffering from the disease and have been evaluated in early clinical trials. Additional antibodies directed against leukocyte integrin components such as etrolizumab (targeting $\beta 7$ integrin as well as E-cadherin) [81] or PF-00547,659 (targeting endothelial MAdCAM-1) [82] are currently being evaluated. Tofacitinib is a small molecule selectively inhibiting Janus kinase (JAK) 1 and 3 over JAK2 that can be administered orally. Blockade of the JAK inhibits several pathways integral to lymphocyte activation, function, and proliferation, including interleukins $2,4,7,9,15$, and 21, thereby influencing both innate and adaptive immune responses [83]. While showing a tolerable safety profile in UC patients, tofacitinib achieved a favorable clinical response rate at week 8 of up to $78 \%$ as compared to $42 \%$ in 


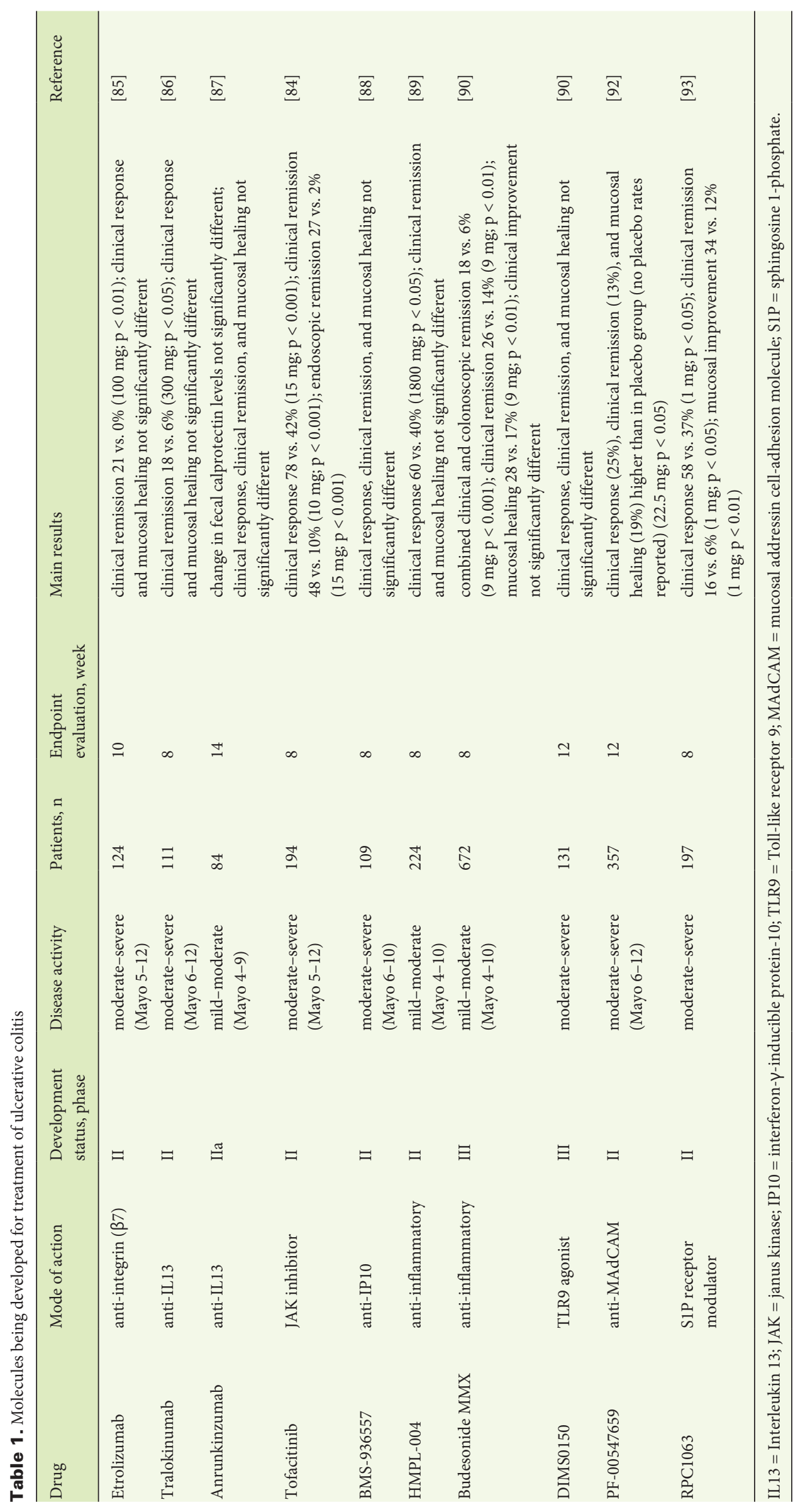


placebo-treated patients [84]. The most important modes of action of new drugs in the treatment of UC are reduction of T cell activation, blockade of cell adhesions molecules, and targeting of proinflammatory cytokines. Molecules currently being developed for the treatment of UC are summarized in table 1.

Concerning the increasing drug armamentarium in UC, direct head-to-head studies of new agents comparing them with existing therapies would advance the care of our patients and enable the best strategy to be selected in order to achieve better outcomes. Furthermore, it is important to identify predictive parameters for treatment response with different agents in individual patients.

\section{Disclosure Statement}

A. Stallmach has served as a board member for Abbvie, Hospira, MSD, Jansen, and Takeda and has received speaker honoraria from Abbvie, FALK HLR, MSD, Recordati Pharma, and Takeda.

C. Schmidt has received speaker honoraria from Abbvie, MSD, and Takeda.

N. Teich has served as a board member for Abbvie, MSD, and Takeda and has received speaker honoraria from Abbvie, MSD, and Takeda.

M. Bürger has no conflicts of interest to disclose.

\section{References}

1 Solberg IC, Lygren I, Jahnsen J, et al: Clinical course during the first 10 years of ulcerative colitis: results from a population-based inception cohort (IBSEN Study). Scand J Gastroenterol 2009;44:431-440.

2 Langholz E, Munkholm P, Davidsen M, et al: Changes in extent of ulcerative colitis: a study on the course and prognostic factors. Scand J Gastroenterol 1996;31: 260-266.

3 Burisch J, Pedersen N, Cukovic-Cavka S, et al.; EpiCom Group: Initial disease course and treatment in an inflammatory bowel disease inception cohort in Europe: the ECCO-EpiCom cohort. Inflamm Bowel Dis 2014;20:36-46.

4 Sandborn WJ: State-of-the-art: immunosuppression and biologic therapy. Dig Dis 2010;28:536-542.

5 Blonski W, Buchner AM, Lichtenstein GR: Treatment of ulcerative colitis. Curr Opin Gastroenterol 2014;30: 84-96.

6 Lissner D, Siegmund B: Ulcerative colitis: current and future treatment strategies. Dig Dis 2013;31:91-94.

7 Dignass A, Lindsay JO, Sturm A, et al: Second European evidence-based consensus on the diagnosis and management of ulcerative colitis part 2: current management. J Crohns Colitis 2012;6:991-1030.

8 Munkholm P, Michetti P, Probert CS, et al: Best practice in the management of mild-to-moderately active ulcerative colitis and achieving maintenance of remission using mesalazine. Eur J Gastroenterol Hepatol 2010;22:912-916.

9 Higgins PD, Rubin DT, Kaulback K, et al: Systematic review: impact of non-adherence to 5-aminosalicylic acid products on the frequency and cost of ulcerative colitis flares. Aliment Pharmacol Ther 2009;29:247-257.

10 Cerveny P, Bortlík M, Kubena A, et al: Nonadherence in inflammatory bowel disease: results of factor analysis. Inflamm Bowel Dis 2007;13:1244-1249.

11 Truelove SC, Witts LJ: Cortisone in ulcerative colitis final report on a therapeutic trial. Br Med J 1955;2: 1041-1048.

12 Marshall JK, Thabane M, Steinhart AH, et al: Rectal 5 -aminosalicylic acid for maintenance of remission in ulcerative colitis. Cochrane Database Syst Rev 2012; 11:CD004118.

13 Andus T, Kocjan A, Müser M, et al: Clinical trial: novel high-dose $1 \mathrm{~g}$ mesalamine suppository (Salofalk) once daily is as efficacious as a $500-\mathrm{mg}$ suppository thrice daily in active ulcerative proctitis. Inflamm Bowel Dis 2010;16:1947-1956

14 Marshall JK, Irvine EJ: Rectal corticosteroids versus alternative treatments in ulcerative colitis: a meta-analysis. Gut 1997;40:775-781.
15 Regueiro M, Loftus EV Jr, Steinhart AH, et al: Medical management of left-sided ulcerative colitis and ulcerative proctitis: critical evaluation of therapeutic trials. Inflamm Bowel Dis 2006;12:979-994.

16 Safdi M, DeMicco M, Sninsky C, et al: A double-blind comparison of oral versus rectal mesalamine versus combination therapy in the treatment of distal ulcerative colitis. Am J Gastroenterol 1997;92:1867-1871.

17 Mulder CJ, Fockens P, Meijer JW, et al: Beclomethasone dipropionate $(3 \mathrm{mg}$ ) versus 5 -aminosalicylic acid $(2 \mathrm{~g})$ versus the combination of both $(3 \mathrm{mg} / 2 \mathrm{~g})$ as retention enemas in active ulcerative proctitis. Eur J Gastroenterol Hepatol 1996;8:549-553.

18 Ford AC, Khan KJ, Achkar JP, Moayyedi P: Efficacy of oral vs. topical, or combined oral and topical 5-aminosalicylates, in ulcerative colitis: systematic review and meta-analysis. Am J Gastroenterol 2012;107:167-176; author reply 177

19 Pimpo MT, Galletti B, Palumbo G, et al: Mesalazine vanishing time from rectal mucosa following its topical administration. J Crohns Colitis 2010;4:102-105.

20 Frieri G, Giacomelli R, Pimpo M, et al: Mucosal 5-aminosalicylic acid concentration inversely correlates with severity of colonic inflammation in patients with ulcerative colitis. Gut 2000;47:410-414.

21 Cortot A, Maetz D, Degoutte E, et al: Mesalamine foam enema versus mesalamine liquid enema in active left-sided ulcerative colitis. Am J Gastroenterol 2008; 103:3106-3114.

22 Eliakim R, Tulassay Z, Kupcinskas L, et al: Clinical trial: randomized-controlled clinical study comparing the efficacy and safety of a low-volume vs. a high-volume mesalazine foam in active distal ulcerative colitis. Aliment Pharmacol Ther 2007;26:1237-1249.

23 Manguso F, Balzano A: Meta-analysis: the efficacy of rectal beclomethasone dipropionate vs. 5-aminosalicylic acid in mild to moderate distal ulcerative colitis. Aliment Pharmacol Ther 2007;26:21-29.

24 Kamm MA, Sandborn WJ, Gassull M, et al: Oncedaily, high-concentration MMX mesalamine in active ulcerative colitis. Gastroenterology 2007;132:66-75; quiz 432-433.

25 Kruis W, Kiudelis G, Rácz I, et al: Once daily versus three times daily mesalazine granules in active ulcerative colitis: a double-blind, double-dummy, randomised, non-inferiority trial. Gut 2009;58:233-240.

26 Ford AC, Achkar JP, Khan KJ, et al: Efficacy of 5-aminosalicylates in ulcerative colitis: systematic review and meta-analysis. Am J Gastroenterol 2011;106:601-616.

27 Hanauer SB, Sandborn WJ, Kornbluth A, et al: Delayed-release oral mesalamine at $4.8 \mathrm{~g} /$ day $(800 \mathrm{mg}$ tablet) for the treatment of moderately active ulcerative colitis: the ASCEND II trial. Am J Gastroenterol 2005;100:2478-2485.
28 Sutherland L, Macdonald JK: Oral 5-aminosalicylic acid for induction of remission in ulcerative colitis. Cochrane Database Syst Rev 2006;CD000543.

29 Feagan BG, Macdonald JK: Oral 5-aminosalicylic acid for induction of remission in ulcerative colitis. Cochrane Database Syst Rev 2012;10:CD000543.

30 Sandborn WJ, Kamm MA, Lichtenstein GR, et al: MMX Multi Matrix System mesalazine for the induction of remission in patients with mild-to-moderate ulcerative colitis: a combined analysis of two randomized, double-blind, placebo-controlled trials. Aliment Pharmacol Ther 2007;26:205-215.

31 Jakobovits SL, Travis SP: Management of acute severe colitis. Br Med Bull 2005;75-76:131-44.

32 Truelove SC, Jewell DP: Intensive intravenous regimen for severe attacks of ulcerative colitis. Lancet 1974;1: 1067-1070.

33 Turner D, Walsh CM, Steinhart AH, et al: Response to corticosteroids in severe ulcerative colitis: a systematic review of the literature and a meta-regression. Clin Gastroenterol Hepatol 2007;5:103-110.

34 Baron JH, Connell AM, Kanaghinis TG, et al: Outpatient treatment of ulcerative colitis. Comparison between three doses of oral prednisone. Br Med J 1962; 2:441-443.

35 Tøttrup A, Erichsen R, Sværke C, et al: Thirty-day mortality after elective and emergency total colectomy in Danish patients with inflammatory bowel disease: a population-based nationwide cohort study. BMJ Open 2012;2:e000823.

36 Lindgren SC, Flood LM, Kilander AF, et al: Early predictors of glucocorticosteroid treatment failure in severe and moderately severe attacks of ulcerative colitis. Eur J Gastroenterol Hepatol 1998;10:831-835.

37 Manosa M, Flood LM, Kilander AF, et al: Decision tree for early introduction of rescue therapy in active ulcerative colitis treated with steroids. Inflamm Bowel Dis 2011;17:2497-2502

38 Ho GT, Mowat C, Goddard CJ, et al: Predicting the outcome of severe ulcerative colitis: development of a novel risk score to aid early selection of patients for second-line medical therapy or surgery. Aliment Pharmacol Ther 2004;19:1079-1087.

39 Lichtiger S, Present DH, Kornbluth A, et al: Cyclosporine in severe ulcerative colitis refractory to steroid therapy. N Engl J Med 1994;330:1841-1845.

40 Van Assche G, D'Haens G, Noman M, et al: Randomized, double-blind comparison of $4 \mathrm{mg} / \mathrm{kg}$ versus $2 \mathrm{mg} / \mathrm{kg}$ intravenous cyclosporine in severe ulcerative colitis. Gastroenterology 2003;125:1025-1031.

41 Bruns T, Schmidt C, Stallmach A: Calcineurin inhibitors or anti-TNF-alpha agents in severe ulcerative colitis: available options and limitations. Z Gastroenterol 2012;50:396-406. 
42 Baumgart DC, Macdonald JK, Feagan B: Tacrolimus (FK506) for induction of remission in refractory ulcerative colitis. Cochrane Database Syst Rev 2008; CD007216.

43 Schmidt KJ, Herrlinger KR, Emmrich J, et al: Shortterm efficacy of tacrolimus in steroid-refractory ulcerative colitis - experience in 130 patients. Aliment Pharmacol Ther 2013;37:129-136.

44 Miyoshi J, Matsuoka K, Inoue N, et al: Mucosal healing with oral tacrolimus is associated with favorable medium- and long-term prognosis in steroid-refractory/ dependent ulcerative colitis patients. J Crohns Colitis 2013; 7:e609-614.

45 Protic M, Seibold F, Schoepfer A, et al: The effectiveness and safety of rescue treatments in 108 patients with steroid-refractory ulcerative colitis with sequential rescue therapies in a subgroup of patients. J Crohns Colitis 2014;8:1427-1437.

46 Kawakami K, Inoue T, Murano M, et al: Effects of ora tacrolimus as a rapid induction therapy in ulcerative colitis. World J Gastroenterol 2015;21:1880-1886.

47 Stack WA, Long RG, Hawkey CJ: Short- and long-term outcome of patients treated with cyclosporin for severe acute ulcerative colitis. Aliment Pharmacol Ther 1998; 12:973-978.

48 Arts J, D’Haens G, Zeegers M, et al: Long-term outcome of treatment with intravenous cyclosporin in patients with severe ulcerative colitis. Inflamm Bowel Dis 2004;10:73-78.

49 Holme O, Thiis-Evensen E, Vatn MH: Treatment of fulminant ulcerative colitis with cyclosporine A. Scand J Gastroenterol 2009;44:1310-1314

50 Sharkey L, Bredin F, Nightingale A, Parkes M: The use of Cyclosporin A in acute steroid-refractory ulcerative colitis: long term outcomes. J Crohns Colitis 2011;5: 91-94.

51 Walch A, Meshkat M, Vogelsang H, et al: Long-term outcome in patients with ulcerative colitis treated with intravenous cyclosporine $\mathrm{A}$ is determined by previous exposure to thiopurines. J Crohns Colitis 2010;4:398404.

52 Actis GC, Fadda M, David E, Sapino A: Colectomy rate in steroid-refractory colitis initially responsive to $\mathrm{cy}$ closporin: a long-term retrospective cohort study. BMC Gastroenterol 2007;7:13.

53 Jarnerot G, Hertervig E, Friis-Liby I, et al: Infliximab as rescue therapy in severe to moderately severe ulcerative colitis: a randomized, placebo-controlled study. Gastroenterology 2005;128:1805-1811.

54 Lees CW, Heys D, Ho GT, et al: A retrospective analysis of the efficacy and safety of infliximab as rescue therapy in acute severe ulcerative colitis. Aliment Pharmacol Ther 2007;26:411-419.

55 Mortensen C, Caspersen S, Christensen NL, et al: Treatment of acute ulcerative colitis with infliximab, a retrospective study from three Danish hospitals. J Crohns Colitis 2011;5:28-33.

56 Kohn A, Daperno M, Armuzzi A, et al: Infliximab in severe ulcerative colitis: short-term results of different infusion regimens and long-term follow-up. Aliment Pharmacol Ther 2007;26:747-756.

57 Laharie D, Bourreille A, Branche J, et al: Ciclosporin versus infliximab in patients with severe ulcerative colitis refractory to intravenous steroids: a parallel, openlabel randomised controlled trial. Lancet 2012;380: 1909-1915.

58 Lowenberg M, Duijvis NW, Ponsioen C, et al: Length of hospital stay and associated hospital costs with infliximab versus cyclosporine in severe ulcerative colitis. Eur J Gastroenterol Hepatol 2014;26:1240-1246.
59 Laharie D, Bourreille A, Branche J, et al: Long-term outcomes in a cohort of patients with acute severe ulcerative colitis refractory to intravenous steroids treated with cyclosporine or infliximab. ECCO 2015; abstr OP017.

60 Naves JE, Llaó J, Ruiz-Cerulla A, et al: Long-term comparative efficacy of cyclosporine- or infliximab-based strategies for the management of steroid-refractory ulcerative colitis attacks. Inflamm Bowel Dis 2014;20: 1375-1381.

61 Armuzzi A, Pugliese D, Danese S, et al: Long-term combination therapy with infliximab plus azathioprine predicts sustained steroid-free clinical benefit in steroid-dependent ulcerative colitis. Inflamm Bowel Dis 2014;20:1368-1374

62 Lequoy M, Seksik P, Bouhnik Y, et al: Efficacy of adalimumab in refractory ulcerative colitis in clinical practice. ECCO 2013;abstr P516.

63 Thorlund K, Druyts E, Toor K, Mills EJ: Comparative efficacy of golimumab, infliximab, and adalimumab for moderately to severely active ulcerative colitis: a network meta-analysis accounting for differences in trial designs. Expert Rev Gastroenterol Hepatol 2015;9:1-8.

64 Gan SI, Beck PL: A new look at toxic megacolon: an update and review of incidence, etiology, pathogenesis, and management. Am J Gastroenterol 2003;98:2363-2371.

65 Grainge MJ, West J, Card TR: Venous thromboembolism during active disease and remission in inflammatory bowel disease: a cohort study. Lancet 2010;375: 657-663.

66 Takeuchi K, Smale S, Premchand P, et al: Prevalence and mechanism of nonsteroidal anti-inflammatory drug-induced clinical relapse in patients with inflammatory bowel disease. Clin Gastroenterol Hepatol 2006;4:196-202.

67 Dickinson RJ, O'Connor HJ, Pinder I, et al: Double blind controlled trial of oral vancomycin as adjunctive treatment in acute exacerbations of idiopathic colitis. Gut 1985;26:1380-1384

68 Mantzaris GJ, Hatzis A, Kontogiannis P, Triadaphyllou G: Intravenous tobramycin and metronidazole as an adjunct to corticosteroids in acute, severe ulcerative colitis. Am J Gastroenterol 1994;89:43-46.

69 Chapman RW, Selby WS, Jewell DP: Controlled trial of intravenous metronidazole as an adjunct to corticosteroids in severe ulcerative colitis. Gut 1986;27:1210-1212.

70 Lobaton T, Vermeire S, Van Assche G, Rutgeerts P: Review article: anti-adhesion therapies for inflammatory bowel disease. Aliment Pharmacol Ther 2014;39: 579-594.

71 Milch C, Wyant T, Xu J, et al: Vedolizumab, a monoclonal antibody to the gut homing alpha4beta7 integrin, does not affect cerebrospinal fluid T-lymphocyte immunophenotype. J Neuroimmunol 2013;264:123-126.

72 Feagan BG, Rutgeerts P, Sands BE, et al.; GEMINI 1 Study Group: Vedolizumab as induction and maintenance therapy for ulcerative colitis. N Engl J Med 2013; 369:699-710

73 Rutgeerts P, Sandborn WJ, Feagan BG, et al: Infliximab for induction and maintenance therapy for ulcerative colitis. N Engl J Med 2005;353:2462-2476.

74 Duboc H, Rajca S, Rainteau D, et al: Connecting dysbiosis, bile-acid dysmetabolism and gut inflammation in inflammatory bowel diseases. Gut 2013;62:531-539.

75 Hold GL, Smith M, Grange C, et al: Role of the gut microbiota in inflammatory bowel disease pathogenesis: what have we learnt in the past 10 years? World J Gastroenterol 2014;20:1192-1210.

76 Angelberger S, Reinisch W, Makristathis A, et al: Temporal bacterial community dynamics vary among ulcerative colitis patients after fecal microbiota transplantation. Am J Gastroenterol 2013;108:1620-1630.
77 Chen WX, Ren LH, Shi RH: Enteric microbiota leads to new therapeutic strategies for ulcerative colitis. World J Gastroenterol 2014;20:15657-15663.

78 Bürger M, Lange K, Stallmach A: Intestinales Mikrobiom und chronisch-entzündliche Darmerkrankungen: Feindschaft oder Freundschaft? Gastroenterologe 2015;10:87-101.

79 Moayyedi P, Surette MG, Kim PT, Libertucci J, Wolfe M, Onischi C, Armstrong D, Marshall JK, Kassam Z, Reinisch W, Lee CH: Fecal microbiota transplantation induces remission in patients with active ulcerative colitis in a randomized controlled trial. Gastroenterology 2015;149:102-109

80 Rossen NG, Fuentes S, van der Spek MJ, Tijssen JG Hartman JH, Duflou A, Löwenberg M, van den Brink GR, Mathus-Vliegen EM, de Vos WM, Zoetendal EG, D'Haens GR, Ponsioen CY: Findings from a randomized controlled trial of fecal transplantation for patients with ulcerative colitis. Gastroenterology 2015;149:110-118.

81 Rutgeerts PJ, Fedorak RN, Hommes DW, et al: A randomised phase I study of etrolizumab (rhuMAb beta7) in moderate to severe ulcerative colitis. Gut 2013;62: 1122-1130.

82 Vermeire S, Ghosh S, Panes J, et al: The mucosal addressin cell adhesion molecule antibody PF-00547,659 in ulcerative colitis: a randomised study. Gut 2011;60 1068-1075.

83 Coskun M, Salem M, Pedersen J, Nielsen OH: Involvement of JAK/STAT signaling in the pathogenesis of inflammatory bowel disease. Pharmacol Res 2013;76:1-8.

84 Sandborn WJ, Ghosh S, Panes J, et al: Tofacitinib, an oral Janus kinase inhibitor, in active ulcerative colitis. N Engl J Med 2012;367:616-624.

85 Vermeire S, O’Byrne S, Keir M, et al: Etrolizumab as induction therapy for ulcerative colitis: a randomised, controlled, phase 2 trial. Lancet 2014;384:309-318.

86 Danese S, Rudziński J, Brandt W, et al: Tralokinumab for moderate-to-severe UC: a randomised, doubleblind, placebo-controlled, phase IIa study. Gut 2015; 64:243-249.

87 Reinisch W, Panés J, Khurana S, et al: Anrukinzumab, an anti-interleukin 13 monoclonal antibody, in active UC: efficacy and safety from a phase IIa randomised multicentre study. Gut 2015;64:894-900.

88 Mayer L, Sandborn WJ, Stepanov Y, et al: Anti-IP-10 antibody (BMS-936557) for ulcerative colitis: a phase II randomised study. Gut 2014;63:442-450.

89 Sandborn WJ, Targan SR, Byers VS, et al: Andrographis paniculata extract (HMPL-004) for active ulcerative colitis. Am J Gastroenterol 2013;108:90-98.

90 Sandborn WJ, Danese S, D'Haens G, et al: Induction of clinical and colonoscopic remission of mild-to-moderate ulcerative colitis with budesonide MMX $9 \mathrm{mg}$ pooled analysis of two phase 3 studies. Aliment Pharmacol Ther 2015;41:409-418.

91 Atreya R, Bloom S, Scaldaferri F, et al: OP015 Multicentre clinical trial with topical administration of the Toll-Like receptor 9 agonist DIMS0150 shows evidence for efficacy in moderate to severe Ulcerative Colitis. ECCO 2015; abstr OP015.

92 Vermeire S, Sandborn W, Danese S, et al: OP021 TURANDOT: a randomized, multicenter doubleblind, placebo-controlled study of the safety and efficacy of Anti-MAdCAM Antibody PF-00547659 (PF) in patients with moderate to severe Ulcerative Colitis (UC). ECCO 2015; abstr OP021.

93 Sandborn W, Feagan B, Wolf D, et al: OP024 A randomized, double-blind, placebo-controlled induction trial of an oral S1P receptor modulator (RPC1063) in moderate to severe Ulcerative Colitis: results of the TOUCHSTONE study. ECCO 2015; abstr OP024. 